\title{
ESCENARIOS EN EL LOGRO DE OBJETIVOS EDUCACIONALES DE ALFABETIZACIÓN INICIAL EN EL NIVEL PREESCOLAR MEXICANO
}

\author{
Ana María Luna Peña \\ Rocío Elizabeth Mancilla Francisco \\ Instituto Noreste (Nuevo León, México)
}

\begin{abstract}
RESUMEN: La educación formal es la respuesta a la necesidad de una práctica educativa ordenada y eficiente. En México, la Secretaría de Educación Pública es la institución encargada de ordenar la práctica educativa en todos los niveles del sistema educativo nacional. El currículo de preescolar, por su parte, ha sido definido por esta desde una perspectiva formativa, que no contempla que el niño egrese leyendo de manera convencional y autónoma. Aun así, las instituciones preescolares privadas de este país suelen solicitar a sus docentes que sean capaces de lograr que el niño egrese leyendo y escribiendo fluidamente. La presente investigación descriptiva ofrece un acercamiento comparativo de los factores implicados en el logro de objetivos educacionales de alfabetización en escenarios preescolares públicos y privados mexicanos. Se apoya en dos instrumentos, aplicados entre febrero y junio de 2018: un cuestionario a docentes y una entrevista a directores; estos cumplen el objetivo de responder: ¿cuál es el grado de percepción de logro que tienen los docentes de preescolares mexicanos de los objetivos educacionales relacionados con la alfabetización inicial de sus alumnos?, y ¿cuáles son las condiciones y requisitos que establecen las escuelas preescolares tanto públicas como privadas para el ejercicio de su práctica docente?
\end{abstract}

PALABRAS CLAVE: alfabetización, educación de la primera infancia, objetivo de enseñanza.

\section{CONTEXTS FOR THE FULFILLMENT OF LITERACY GOALS IN MEXICAN EARLY CHILDHOOD EDUCATION}

\footnotetext{
ABSTRACT: Formal education is the answer to the need of an orderly and efficient teaching practice. In Mexico, the Secretariat of Public Education is the institution mandated to organize the teaching practice at every level. The preschool curriculum has been defined by this institution with a formative
} 
perspective, which does not expect the children to graduate with autonomous and conventional reading competencies at this level. Even so, private preschools in the country commonly ask their teachers to achieve fluid writing and reading skills on their students. This research compares the factors implied in the achievement of literacy goals in public and private schools contexts. This research is supported by two data gathering instruments, applied between February and June 2018: one survey for preschool teachers and one interview with preschool principals; these instruments provide with an answer to the following questions: What is the Mexican preschool teachers' perception of achievement on their students' initial literacy? What are the conditions and requirements set by private and public preschools on their teaching practice?

KEYWORDS: Literacy, early childhood education, teaching objective.

Recibido: 14/01/2019

Aceptado: 28/02/2019

Correspondencia: Ana María Luna Peña. Instituto Noreste. C/ Gral. Francisco Villa 207, Los Elizondo, Cd Gral Escobedo, N.L. (Nuevo León, México). Email: analuna@inieam.org.mx.

\section{INTRODUCCIÓN}

La educación es un proceso fundamental en la vida de todos los seres humanos: inicia incluso antes del nacimiento y abarca todas las etapas de desarrollo, dotando a los individuos de aprendizajes y competencias útiles para la existencia individual y colectiva. Y si bien la educación no depende de las aulas escolares, pues tanto niños como adultos aprenden todo el tiempo, en diferentes contextos y a lo largo de la vida, es en la educación formal -es decir, la que se imparte institucionalmente "dentro y/o fuera de sus instalaciones físicas" (Arnaz, 1990, p. 68)-, en la que se busca el desarrollo completo de la persona a través de acciones sistemáticas y ordenadas, casi siempre ausentes en la educación informal. Es más, la disciplina de la Didáctica, por no decir de la Pedagogía moderna, comenzó cuando Jan Amós Comenio (2006) argumentó que "no requiere otra cosa el arte de enseñar que una ingeniosa disposición del tiempo, los objetos y el método" (p. 51).

Desafortunadamente en el nivel de educación preescolar, que "cumple funciones económicas, sociopolíticas y pedagógicas [...], en repetidas ocasiones, se le ha dado prioridad a las dos primeras en detrimento de la función pedagógica" (Chaves Salas, 2002 , p. 3). Lo cual es lamentable, ya que es la parte pedagógica -específicamente las técnicas y las estrategias de enseñanza- la que se convierte en la verdadera herramienta para lograr los objetivos de este nivel de educación. Pese a esto, las funciones económicas y sociopolíticas pueden llegar a perturbar el objetivo pedagógico declarado por la ley, la teoría y la práctica.

Esta investigación nació de la necesidad de revalorar la función pedagógica del nivel de preescolar mexicano, por encima de las necesidades económicas y merca- 
dotécnicas que parecen abrir una brecha entre la educación pública y la educación privada en este país. Específicamente la investigación se enfocó en descubrir los escenarios en que sucede la búsqueda del logro de objetivos educacionales de alfabetización inicial en preescolares mexicanos y a responder las siguientes preguntas: ¿cuál es el grado de percepción de logro que tienen los docentes de preescolares mexicanos de los objetivos educacionales relacionados con la alfabetización inicial de sus alumnos?, y ¿cuáles son las condiciones y requisitos que establecen las escuelas preescolares tanto públicas como privadas para el ejercicio de su práctica docente?

Se usará el término de objetivos educacionales -en el sentido otorgado por Arnaz (1990) de "meta, enunciado con el que se describe un propósito en materia de educación, esto es, la finalidad por la que se emprenden determinados esfuerzos" (p. 69) - para referir al conjunto de aprendizajes esperados, objetivos de egreso, competencias terminales, que a lo largo de la historia han integrado el currículum de preescolar del sistema educativo mexicano.

\section{FundAMENTACIÓN TEÓRICA}

Las instituciones escolares no siempre han sido como lo son ahora, ni son iguales en las diferentes poblaciones del mundo, a pesar de que en ellas, por su misma definición, se imparta la educación formal. No obstante, reflejan la preocupación de la sociedad no solo por atender el derecho a la educación de sus miembros, sino por procurar su pleno desarrollo a través de una educación ordenada y sistemática. Y es que organizar la práctica educativa según las características de cada sociedad es también una necesidad universal, como la de la misma educación. Ya en tiempos prehispánicos, en el territorio mexicano, en los telpochcalli y calmécac se procuraba la formación de los jóvenes mexicas, como mencionan Solana, Cardiel Reyes y Bolaños (2001). Dichos autores también documentan la apertura de colegios y universidades durante el periodo colonial de la Nueva España y la determinación tanto de los gobiernos virreinales como de los independientes de establecer la instrucción pública.

Pero es precisamente en las diferencias entre sistemas educativos de épocas o regiones distintas, o bien incluso entre escuelas de una misma época y lugar, en las que se pueden apreciar las necesidades de carácter pedagógico o didáctico, de carácter social o cultural o de carácter económico o político de la sociedad a que corresponden. Para Narodowski y Martínez Bloom (2016), la expansión en los últimos años de la educación privada -o de la privatización de la educación- se trata de una estructura al mismo tiempo política y económica. Lo clarifican por medio del siguiente caso:

Imaginemos, por ejemplo, qué ocurriría si todas las familias de clases medias que mandan a sus hijos a escuelas privadas decidieran inscribirlos en una escuela pública haciendo uso del derecho que les garantiza el ordenamiento jurídico. No caben dudas que no pocos sistemas educativos colapsarían de inmediato por falta de recursos: edificios, vacantes, dinero para pagar los salarios de los nuevos docentes, etc. (p. 22).

La historia anterior no es ajena a los mexicanos: durante el mandato de Venustiano Carranza, poco después de la promulgación de la Constitución Política de 1917, "la 
incapacidad económica y técnica de los municipios para satisfacer las necesidades elementales de la población escolar obligó al Gobierno, según el mismo Carranza, a solicitar colaboración de la iniciativa privada para que ésta cooperara en el servicio educativo" (Solana, Cardiel Reyes y Bolaños, 2001, p. 152). Y si bien este no es el origen de la educación pública y de la privada en México, es relevante mencionarlo, ya que constituye un parteaguas en la historia de su educación, sobre todo porque fueron precisamente dichas insuficiencias las que llevaron a la creación, en 1921, del organismo gubernamental denominado Secretaría de Educación Pública, que se encargó a partir de ese momento de la educación rural, del combate al analfabetismo, de la administración escolar y de la capacitación docente, entre otros asuntos (Zárate González, 2015). A partir de ese momento se comenzó a gestar la mayor obra educativa que en el país se hubiera emprendido, cuyo fruto fue el actual sistema educativo mexicano.

La educación formal en México tiene características y reglas específicas dependiendo del origen del presupuesto de la institución en que se imparte, del nivel, subsistema y modalidad de estudios impartidos, de modo que puede haber educación de sostenimiento privado o público (sea este federal, estatal, autónomo, etc.); educación básica, media superior, superior o de formación para el trabajo; educación general o técnica; o educación escolarizada, no escolarizada o mixta.

El nivel preescolar, por su parte, pertenece a la educación básica y tiene como objetivo preparar a sus alumnos para su ingreso al nivel de la educación primaria, y, al mismo tiempo, formarlos con conocimientos básicos y competencias para la vida, capaces de desarrollar aptitudes, como ser independientes, sociales y autónomos. La educación en este nivel es eminentemente formativa. Oficialmente se define como:

El primer nivel de la educación básica. Busca el desarrollo integral y equilibrado que facilita la relación de los niños con sus pares y con adultos. Promueve la socialización y la afectividad, las capacidades de comunicación, el pensamiento matemático, el conocimiento de los entornos natural y social, el desarrollo y enriquecimiento físico y psicomotriz, y la expresión artística (Modelo educativo para la educación obligatoria, 2017, p. 77).

El Gobierno Federal autoriza a los particulares (personas físicas o morales) a impartir educación preescolar, siempre y cuando cumplan con lo solicitado por la autoridad al respecto de "I. Personal directivo y docente, y II. Instalaciones en las que se impartirá la educación preescolar, las cuales deberán satisfacer las condiciones higiénicas, de seguridad y pedagógicas" (Acuerdo 357, 2005, p. 14).

El Acuerdo Secretarial 357, vigente a la fecha, describe las características obligatorias estipuladas por la Secretaría de Educación Pública que deben reunir los preescolares privados para poder impartir esos estudios con el Reconocimiento de Validez Oficial. Y, si bien su mayor énfasis recae en la obligatoriedad de posesión de una planta docente capacitada e instalaciones apropiadas, se contempla también "el cumplimiento del Programa de Educación Preescolar determinado de conformidad con el artículo 3o. de la Constitución Política de los Estados Unidos Mexicanos y publicado en el Diario Oficial de la Federación" (p. 21). Sin embargo, el Acuerdo no especifica si se puede o no añadir más contenidos a dicho programa. 


\section{LA IMPORTANCIA DE LA EDUCACIÓN PREESCOLAR EN EL DESARROLLO DEL NIÑO}

Por mucho tiempo las ideas al respecto de la educación de la primera infancia fueron descuidadas, pues, en un principio, cuando surgieron los centros infantiles en el mundo contemporáneo, estos "surgieron únicamente como guarderías, lugares donde se cuidaba a los infantes mientras sus madres trabajaban" (Chaves Salas, 2002, p. 3). Sin embargo, según esta misma autora, "posteriormente, gracias a las investigaciones científicas y al desarrollo de la psicología y la pedagogía, se toma conciencia de su valor educativo" (p. 3).

De acuerdo con Braslavsky (2004), antes del siglo XX ya se conocían aportaciones a la educación infantil: Comenio, cuya pedagogía se fundamenta en el desarrollo humano; Rousseau, crítico del "adulto en miniatura" en que querían convertir al niño los educadores de su tiempo; y Pestalozzi, quien también proponía educar al niño según sus "facultades". A ellos se suman en el siglo XX María Montessori, con su casa dei bambini, y Piaget y Vigotsky, dos de los grandes exponentes de la psicología y la pedagogía de la infancia. Otros autores mencionan también a Friedrich Fröbel, creador del kindergarten original; a Estefanía Castañeda Núñez, fundadora del primer jardín de niños en México; y a Ovide Decroly, cuyo método experimental procura educar dentro de un medio natural (Ramos Valdez, 2007).

Por su parte, fue labor de Piaget elaborar una teoría de estadios cognitivos, los cuales "se pueden considerar como una plantilla para determinar los procesos de pensamiento de los alumnos" (Ganem Alarcón y Ragasol Álvarez, 2015, p. 49). Estos estadios -sensoriomotriz o preoperacional, de las operaciones concretas y de las operaciones abstractas- están relacionados "con las capacidades mentales que posee el sujeto para organizar la información que recibe del medio" (Chaves Salas, 2002, p. 10). Para Piaget, "el pensamiento del párvulo surge, entonces, a través de la acción, a partir de la cual interioriza ciertas imágenes que posteriormente, aprenderá que tienen correspondencia con un nombre, y de esa manera, se origina el lenguaje" (Chaves Salas, 2002, p. 10).

Vigotsky, por otra parte, fue el encargado de elaborar la teoría que diera especial atención al proceso histórico-cultural por el cual se desarrollan en el ser humano los procesos psicológicos elementales y los superiores. A esta teoría adscribió tres principios elementales: el de la sociogénesis del desarrollo de procesos superiores (por interacción con el entorno o grupo social), el principio de internalización de estos procesos por el niño (individualización de experiencias) y "el principio activo de la conducta" (Braslavsky, 2004, p. 45).

De acuerdo con esta teoría, el lenguaje se da, en un principio en el nivel social, luego es egocéntrico, y más adelante, interiorizado (Vigotsky, 1978). Cuando el párvulo inicia la comunicación verbal, el lenguaje sigue a las acciones, es provocado y dominado por la actividad; en estadios superiores surge una nueva relación entre la palabra y la acción, ya que el lenguaje guía, determina y domina el curso de la acción y aparece su función planificadora (Chaves Salas, 2002, p. 12). 
Las teorías de estos dos psicólogos a menudo se enfrentan, pero en la gran mayoría de las ocasiones se complementan. Así lo entienden diversos autores ya citados en el presente artículo; por ejemplo, tanto Chaves Salas (2002) como Ganem Alarcón y Ragasol Álvarez (2015) enfatizan que ambos autores lograron un cambio epistemológico en la concepción de los actores del proceso de enseñanza-aprendizaje, al concebirlo como un proceso constructivo; y Braslavsky (2004) recalca en varias ocasiones que afirmar de manera absoluta una postura teórica sobre otra no ayuda a mejorar los problemas relacionados con la enseñanza, específicamente de la alfabetización inicial, sino que se debe procurar un enfoque interdisciplinario.

¿Pero cómo se integran las teorías de estos autores a la práctica del docente? La respuesta está presente en la siguiente idea de Kenneth Goodman (2003): "Los programas de instrucción en la escuela deben planearse teniendo en cuenta el conocimiento que los niños ya tienen" (p. 79). Es decir, que el fin de la escuela es el de darle continuidad a los aprendizajes con los que el niño ya cuenta, construidos en su interacción previa con el medio.

\section{El papel de la alfabetización en los Programas de Estudio de Preescolar}

La educación formal preescolar en México tiene una de sus bases en la apertura, en 1904, "de dos jardines de niños en la ciudad de México: el Federico Fröebel [...] y el Enrique Pestalozzi" (Solana et al., 2001, p. 152). Si bien en otros estados ya existían antecedentes notables, el establecimiento de estos dos institutos consolidó al sistema de jardines de niños en el país durante los años porfiristas. Sin embargo, este nivel educativo comenzó a ser obligatorio a nivel federal solo a partir de la reforma al artículo 3. ${ }^{\circ}$ del año 2002 (Acuerdo 348, 2004). A dicha reforma le siguió un rediseño al Programa de Estudios de Preescolar que se había estado Ilevando hasta entonces, el cual finalmente se publicó en el Diario Oficial de la Federación del 27 de octubre de 2004. El Programa del año 2004 fue organizado en seis campos formativos, siendo Lenguaje y comunicación uno de ellos. En la descripción de este campo formativo se puede apreciar la alta prioridad que se le da al lenguaje oral. Además, aclara que no es el objetivo de la educación preescolar el enseñar a los niños a leer y escribir de manera convencional.

El siguiente rediseño al Programa corresponde al publicado en el Diario Oficial de la Federación del 19 de agosto de 2011 (Acuerdo 592, 2011). Integra cuatro campos formativos, incluido el de Lenguaje y comunicación. En el Programa se expresa que "la propuesta pedagógica de preescolar se sustenta en la comprensión de algunas características y funciones del lenguaje escrito" (Acuerdo 592, 2011, p. 25). Como su precedente, tampoco contempla que el alumno egrese leyendo de forma convencional y autónoma.

El último rediseño al Programa de Preescolar ocurrió en 2017 y fue publicado el 29 de junio de 2017 (Acuerdo 07/06/17, 2017). El Programa contempla once rasgos en el perfil de egreso del alumno de educación básica, correspondiendo uno de ellos al de Lenguaje y comunicación. Los aprendizajes esperados para este, en preescolar, 
se dosifican en cuatro organizadores curriculares: oralidad, estudio, literatura y participación social. La aproximación del niño al sistema de escritura es fundamental para el cumplimiento del perfil de egreso anterior, mas no es obligatorio que aprenda a leer convencional y autónomamente (Secretaría de Educación Pública [SEP], 2017).

El campo Lenguaje y comunicación de este Programa contempla dos propósitos:

1. Adquirir confianza para expresarse, dialogar y conversar en su lengua; mejorar su capacidad de escucha y enriquecer su lenguaje oral al comunicarse en situaciones variadas.

2. Desarrollar interés y gusto por la lectura, usar diversos tipos de texto e identificar para qué sirven; iniciarse en la práctica de la escritura y reconocer algunas propiedades del sistema de escritura (SEP, 2017, p. 188).

Los programas descritos anteriormente coinciden en proponer que el niño en preescolar conozca y utilice el sistema de escritura de una lengua aun cuando no esté obligado a usarlo convencionalmente. Rugeiro y Guevara (2015) Ilaman alfabetización inicial a este proceso de familiarización que antecede a la alfabetización formal de la educación primaria.

¿Pero es incumbencia o no de la educación preescolar enseñar a los niños a leer y a escribir de manera convencional? A lo largo de la historia ha existido en México una discrepancia al respecto. De acuerdo con Carime Hagg (Swartz, Shook, Klein y Hagg, 2001), dicha controversia surge no solo a partir del cuestionamiento didáctico sobre la madurez de los niños para este aprendizaje, "también por razones de competencia entre las escuelas (sobre todo las privadas) y por cuestiones de querer demostrar quién tiene el 'mejor' método para trabajar con los niños de esta edad, y quién los prepara mejor para la primaria" (p. 95).

Lamentablemente esta discusión olvida que el niño solo aprenderá a leer y a escribir si lo considera una necesidad, independientemente de que se encuentre inscrito a una institución pública o a una institución privada. Los docentes no deben olvidar esta idea, expresada muy enfáticamente por Vigotsky, en la que se refiere a la necesidad de "empezar a enseñar a leer y escribir en el preescolar, pero se debe enseñar de un modo en que la escritura sea necesaria para el niño, es decir, sentida por él como una necesidad natural" (Braslavsky, 2004, p. 56).

En cualquier caso, sea que se enseñe a leer y escribir o que se inicien las bases de dicho aprendizaje, no hay que olvidar que la escritura y lectura no son cuestiones únicamente escolares.

Como Yetta Goodman dice, generaciones de padres han lavado de las paredes la evidencia del desarrollo de la escritura de sus hijos. La verdad es que los niños Ilegan a la escuela ricos en experiencias de lectoescritura y también ya han aprendido a darle significado a lo impreso (Goodman, 2003, p. 79).

Otros autores respaldan esta propuesta, como Emilia Ferreiro (2000), quien expresa que la lectoescritura es importante por su relevancia social: "Hay que ser enfáticos: la escritura es importante en la escuela porque es importante fuera de la escuela, y 
no al revés" (p. 45). Del mismo modo, Rivas González (2007) está convencida de que "la lectura y la escritura no son propiedad escolar, son producciones sociales propiedad y derecho de todas y todos los seres humanos y por ende de las niñas y los niños" (p. 32).

\section{Métodos de La enSeÑanZa de LA leCtOesCritura}

A continuación, en la Tabla 1 se ofrece una breve síntesis de los métodos en la enseñanza de la lectoescritura, los cuales, según Braslavsky (2014), se dividen en métodos de marcha sintética y métodos de marcha analítica; además, se complementa con información del método ecléctico, aportada por Chaves Salas (2002).

Tabla 1. Clasificación de los métodos de enseñanza de la lectoescritura (Elaboración propia con información de Braslavsky, 2014, pp. $43-60$ y de Chaves Salas, 2002, pp. 8-9)

\begin{tabular}{|c|c|c|}
\hline \multicolumn{3}{|c|}{ Clasificación de los métodos de enseñanza de la lectoescritura } \\
\hline Clasificación general & Clasificación específica & Descripción \\
\hline De marcha sintética & $\begin{array}{l}\text { Método alfabético, de letra, } \\
\text { literal o grafemático }\end{array}$ & Parte de las letras o grafemas \\
\hline \multirow{3}{*}{ De marcha sintética } & Métodos fonéticos o fónicos & Parten de sonidos o fonemas \\
\hline & $\begin{array}{l}\text { Método silábico } \\
\text { Tipo de método fonético }\end{array}$ & $\begin{array}{l}\text { Parte de las sílabas, combi- } \\
\text { nando vocales y consonantes }\end{array}$ \\
\hline & $\begin{array}{l}\text { Método psicofonético } \\
\text { Tipo de método fonético }\end{array}$ & $\begin{array}{l}\text { Parte de las sílabas, presenta- } \\
\text { das gradualmente, en orden } \\
\text { sistemático }\end{array}$ \\
\hline \multirow{4}{*}{ De marcha analítica } & $\begin{array}{l}\text { Métodos naturales, ideovisuales, glo- } \\
\text { bales o globales analíticos }\end{array}$ & $\begin{array}{l}\text { Parten de la palabra, la frase } \\
\text { o el cuento }\end{array}$ \\
\hline & $\begin{array}{l}\text { Método de las frases normales } \\
\text { Tipo de método global }\end{array}$ & Parte de la frase \\
\hline & $\begin{array}{l}\text { Método de la historieta o del cuento } \\
\text { Tipo de método global }\end{array}$ & Parte del cuento \\
\hline & $\begin{array}{l}\text { Métodos de la palabra: método } \\
\text { de la palabra sin análisis y de la pala- } \\
\text { bra generadora } \\
\text { Tipo de método global }\end{array}$ & Parten de la palabra \\
\hline Métodos eclécticos & \multicolumn{2}{|l|}{ Integran métodos sintéticos y analíticos } \\
\hline
\end{tabular}

Las estrategias de aplicación de estos métodos podrán variar dependiendo de variables como el grado de desarrollo cognoscitivo, lingüístico, socioemocional, adaptativo o físico del niño que está aprendiendo la lectoescritura (Ramos y Ramos, 2010); de su tipo de familia (De-La-Peña, Parra-Bolaños y Fernández-Medina, 2018); o de 
la calidad del ambiente letrado y el nivel socioeconómico del instituto al que asiste (Orellana-García y Melo-Hurtado, 2014), entre otras.

\section{Método}

La investigación que a continuación se describe es mixta, pues participa tanto del enfoque cuantitativo como del cualitativo.

En el primer caso se refiere a una investigación de alcance descriptivo, no experimental, cuya técnica de recolección de datos (un cuestionario cerrado) se aplicó en un solo momento. Tuvo como objetivo principal conocer el grado de percepción de logro que tienen los docentes de los objetivos educacionales relacionados con la alfabetización inicial de sus alumnos, tanto en escuelas públicas como privadas. Se complementó este estudio cuantitativo con uno cualitativo, a través de la aplicación de una entrevista estructurada a diversos directivos de escuelas preescolares con el objetivo de conocer las condiciones y requisitos establecidos por la autoridad para el ejercicio de la práctica docente de sus maestros.

El objetivo general del presente estudio es ofrecer un acercamiento comparativo del logro de objetivos educacionales de alfabetización en escenarios preescolares públicos y privados mexicanos.

\section{UNIVERSO DE ESTUDIO}

La población estudiada está compuesta por docentes y directores de preescolares públicos y privados. En el primer caso, la muestra fue de 84 docentes encuestados al azar, presencialmente y en línea, y, en el segundo, de cuatro directores de educación preescolar del estado de Nuevo León, dos de escuelas públicas y dos de instituciones privadas.

\section{INSTRUMENTOS DE RECOLECCIÓN DE DATOS}

Los instrumentos utilizados fueron dos: un cuestionario cerrado, aplicado a docentes, y una entrevista estructurada, a directores.

El cuestionario constó de tres secciones: la primera, dedicada a obtener información personal y profesional del docente (sexo, edad, último grado de estudios acreditado, estudios en curso, años de experiencia en preescolar, grado que impartía, tipo de sostenimiento de la Institución donde laboraba, Estado y municipio donde laboraba y cantidad de alumnos que había en su aula); la segunda, dedicada a obtener información de las estrategias de enseñanza que utilizaba en su práctica docente (¿Se basa usted en el contenido de los libros recomendados por la Institución para alfabetizar? ¿Parte usted de los intereses y saberes previos de los niños para motivar y para propiciar el aprendizaje de la lectoescritura? ¿Reciben los alumnos ayuda en casa para el proceso de alfabetización? ¿Utiliza usted juegos para alfabetizar?); y la tercera, consistente en dos preguntas: “¿Qué objetivos requeridos por la escuela debe usted alcanzar en el grado que imparte al final del curso?" y “¿Qué objetivos cree 
usted alcanzar en la mayoría de sus alumnos al final del curso?". Los docentes disponían de una lista con 18 ítems:

- Que el niño identifique las vocales (O1)

- Que el niño realice trazos básicos (O2)

- Que el niño identifique su nombre (O3)

- Que el niño distinga mayúsculas de minúsculas (vocales) (O4)

- Que el niño garabatee (O5)

- Que el niño simule la lectura (O6)

- Que el niño identifique las consonantes (O7)

- Que el niño lea sílabas (O8)

- Que el niño escriba su nombre (O9)

- Que el niño distinga mayúsculas de minúsculas (consonantes) (O10)

- Que el niño escriba de izquierda a derecha y respete espacios (O11)

- Que el niño deje espacios entre palabras (O12)

- Que el niño lea palabras (O13)

- Que el niño lea oraciones (O14)

- Que el niño lea textos cortos (O15)

- Que el niño escriba palabras (O16)

- Que el niño escriba oraciones (O17)

- Que el niño escriba textos cortos (O18)

Por su parte, la entrevista a los directores indagaba la cantidad de horas de la jornada escolar, los objetivos educacionales relacionados con el aprendizaje de la lectura y escritura y el material bibliográfico que los docentes utilizaban para planear sus clases.

\section{Resultados}

\section{Resultados del estudio cuantitativo con docentes de preescolar}

Durante los meses de febrero a junio de 2018 se aplicaron encuestas a 84 profesores que en ese momento estuvieran impartiendo clases de preescolar, ya fuera en el sistema público o en el privado, en cualquiera de los tres grados de estudios o en entornos multigrado. Su aplicación fue al azar, en persona y en línea. Se aplicaron 41 encuestas a profesores de escuelas públicas y 43 encuestas a profesores de escuelas privadas, de la manera como se explica en la Tabla 2. 
Tabla 2. Número de docentes que componen la muestra

\begin{tabular}{|l|c|c|c|}
\hline \multicolumn{4}{|c|}{ Número de docentes que componen la muestra } \\
\hline & Sostenimiento público & Sostenimiento privado & Total \\
\hline Primer grado & 3 & 13 & $\mathbf{1 6}$ \\
\hline Segundo grado & 14 & 13 & $\mathbf{2 7}$ \\
\hline Tercer grado & 13 & 16 & $\mathbf{2 9}$ \\
\hline Multigrado & 11 & 1 & $\mathbf{1 2}$ \\
\hline Total & $\mathbf{4 1}$ & $\mathbf{4 3}$ & $\mathbf{8 4}$ \\
\hline
\end{tabular}

Del total de la muestra, un $92.9 \%$ corresponde a mujeres y un $7.1 \%$ corresponde a hombres. Su promedio de edad es de 30.6 años, siendo 19 y 54 los extremos. Con respecto al origen geográfico de los encuestados, $79.8 \%$ trabaja en escuelas del estado de Nuevo León (principalmente de la zona metropolitana de Monterrey) y $20.2 \%$ trabaja en escuelas de otros estados de la república. Con respecto a su nivel de estudios, un 31\% tiene estudios de Bachillerato o de Carrera técnica (como la de Asistente educativo); un 65.5\% cuenta con estudios de Licenciatura o de Normal (sin excluir nivelaciones pedagógicas) y apenas un 3.5\% tiene estudios de Maestría o Doctorado; sin embargo, de la totalidad de docentes encuestados, un $46.4 \%$ se encuentra estudiando algún programa educativo. En relación con la experiencia docente de los encuestados, casi la mitad de la muestra (48.8\%) tiene entre 0 y 4 años de experiencia en el mismo u otro grado de preescolar y un porcentaje significativo (38.1\%) cuenta con 5 a 14 años de experiencia; en cambio, una minoría de los encuestados (8.3\%) cuenta con 15 a 24 años de experiencia y prácticamente ninguno (4.8\%) ha cumplido 25 años de experiencia. El promedio de alumnos por aula es de 20.8, siendo 4 y 35 los extremos. En suma, los docentes encuestados atienden a un total de 1,751 alumnos.

La descripción anterior ofrece las características generales de la muestra; sin embargo, algunos subgrupos de la misma guardan características particulares. Por ejemplo,

- en aulas multigrado no existe una diferencia significativa entre el porcentaje de mujeres y de hombres (66.7\% son mujeres y $33.3 \%$ hombres);

- por otra parte, en aulas privadas la diferencia entre el porcentaje de mujeres y de hombres es absoluta (100\% mujeres y $0 \%$ hombres);

- en aulas multigrado es más probable encontrar docentes que no cuenten aún con el nivel de Licenciatura (91.7\% cuenta con nivel de Bachillerato o carrera técnica y $8.3 \%$ con nivel de Licenciatura);

- en este mismo entorno, la ubicación geográfica de las escuelas donde trabajan dichos docentes es, en su mayoría (75\%), Nuevo León, pero no la zona metropolitana de Monterrey: Anáhuac, Cadereyta Jiménez, García, Mina, Salinas Victoria, Santiago;

- finalmente, el promedio de alumnos por aula difiere significativamente en algunos de los subgrupos de la muestra, variable que podría afectar el desempeño docente. Por su importancia, estas cantidades se detallarán a continuación en la Tabla 3. 
Tabla 3. Promedio de alumnos por aula

\begin{tabular}{|l|c|c|c|}
\hline \multicolumn{4}{|c|}{ Promedio de alumnos por aula } \\
\hline & Sostenimiento público & Sostenimiento privado & Promedio total \\
\hline Primer grado & 17.3 & 18.9 & $\mathbf{1 8 . 1}$ \\
\hline Segundo grado & 26.9 & 19.4 & $\mathbf{2 3 . 2}$ \\
\hline Tercer grado & 27.8 & 21 & $\mathbf{2 4 . 4}$ \\
\hline Multigrado & 8.8 & 30 & $\mathbf{1 9 . 4}$ \\
\hline Promedio total & $\mathbf{2 0 . 2}$ & $\mathbf{2 2 . 3}$ & $\mathbf{2 1 . 3}$ \\
\hline
\end{tabular}

Los docentes encuestados también respondieron cuestionamientos al respecto de sus estrategias de enseñanza, por ejemplo, si implementaban juegos, entre otras. En la Tabla 4 se detallan los datos recabados en estas preguntas.

Tabla 4. Frecuencia de aplicación de estrategias de enseñanza de lectoescritura

\begin{tabular}{|c|c|c|c|c|c|c|}
\hline \multicolumn{7}{|c|}{ Frecuencias de aplicación de estrategias de enseñanza de lectoescritura } \\
\hline \multicolumn{7}{|c|}{ Sostenimiento público } \\
\hline Pregunta & Nunca & $\begin{array}{c}\text { Casi } \\
\text { nunca }\end{array}$ & A veces & $\begin{array}{c}\text { Casi } \\
\text { siempre }\end{array}$ & Siempre & $\begin{array}{c}\text { Otra } \\
\text { respuesta }\end{array}$ \\
\hline $\begin{array}{l}\text { ¿Se basa usted en el contenido } \\
\text { de los libros recomendados por } \\
\text { la Institución para alfabetizar? }\end{array}$ & - & $2.4 \%$ & $24.4 \%$ & $22 \%$ & $46.3 \%$ & $\begin{array}{l}\text { No hay } \\
4.9 \%\end{array}$ \\
\hline $\begin{array}{l}\text { ¿Parte usted de los intereses y } \\
\text { saberes previos de los niños } \\
\text { para motivar y para propiciar el } \\
\text { aprendizaje de la lectoescritura? }\end{array}$ & - & - & - & $29.3 \%$ & $70.7 \%$ & - \\
\hline $\begin{array}{l}\text { ¿Reciben los alumnos ayuda en } \\
\text { casa para el proceso de alfabe- } \\
\text { tización? }\end{array}$ & - & $12.2 \%$ & $56.1 \%$ & $17.1 \%$ & $9.8 \%$ & $\begin{array}{c}\text { Desconoz- } \\
\text { co } \\
4.9 \% \\
\end{array}$ \\
\hline $\begin{array}{l}\text { ¿Utiliza usted juegos para alfa- } \\
\text { betizar? }\end{array}$ & - & - & $19.5 \%$ & $39 \%$ & $41.5 \%$ & - \\
\hline \multicolumn{7}{|c|}{ Sostenimiento privado } \\
\hline $\begin{array}{l}\text { ¿Se basa usted en el contenido } \\
\text { de los libros recomendados por } \\
\text { la Institución para alfabetizar? }\end{array}$ & - & - & $20.9 \%$ & $41.9 \%$ & $37.2 \%$ & - \\
\hline $\begin{array}{l}\text { ¿Parte usted de los intereses y } \\
\text { saberes previos de los niños } \\
\text { para motivar y para propiciar el } \\
\text { aprendizaje de la lectoescritura? }\end{array}$ & - & - & $7 \%$ & $32.6 \%$ & $60.5 \%$ & - \\
\hline $\begin{array}{l}\text { ¿Reciben los alumnos ayuda en } \\
\text { casa para el proceso de alfabe- } \\
\text { tización? }\end{array}$ & - & $2.3 \%$ & $39.5 \%$ & $32.6 \%$ & $16.3 \%$ & $\begin{array}{c}\text { Desconoz- } \\
\text { co } \\
9.3 \%\end{array}$ \\
\hline $\begin{array}{l}\text { ¿Utiliza usted juegos para alfa- } \\
\text { betizar? }\end{array}$ & - & - & $14 \%$ & $44.2 \%$ & $41.9 \%$ & - \\
\hline
\end{tabular}


En cuanto a los métodos que emplean los docentes para enseñar el proceso de lectoescritura, el más usado es el Método silábico, con 65 menciones positivas; le siguieron el Método fónico, con 46; el Método alfabético, con 33; el Método de la palabra o de la palabra generadora, con 22; el Método psicofonético, con 13; el Método global u otros métodos analíticos, con 7; el Método ecléctico, con 7; y Ninguno, con 1. Otros métodos mencionados fueron el "Sensorial", el "Método enseñanzaaprendizaje. Uso de las TICs", el "Alfabeto móvil" y el "Método del Modelo ABCD de CONAFE: Aprender [a] aprender mediante el diálogo y juego".

De acuerdo con la clasificación de estos métodos ofrecida anteriormente (Tabla 1), podemos apreciar que los métodos sintéticos, especialmente los fonéticos, son los más utilizados por los docentes de preescolar en sus aulas de clase (Figura 1).

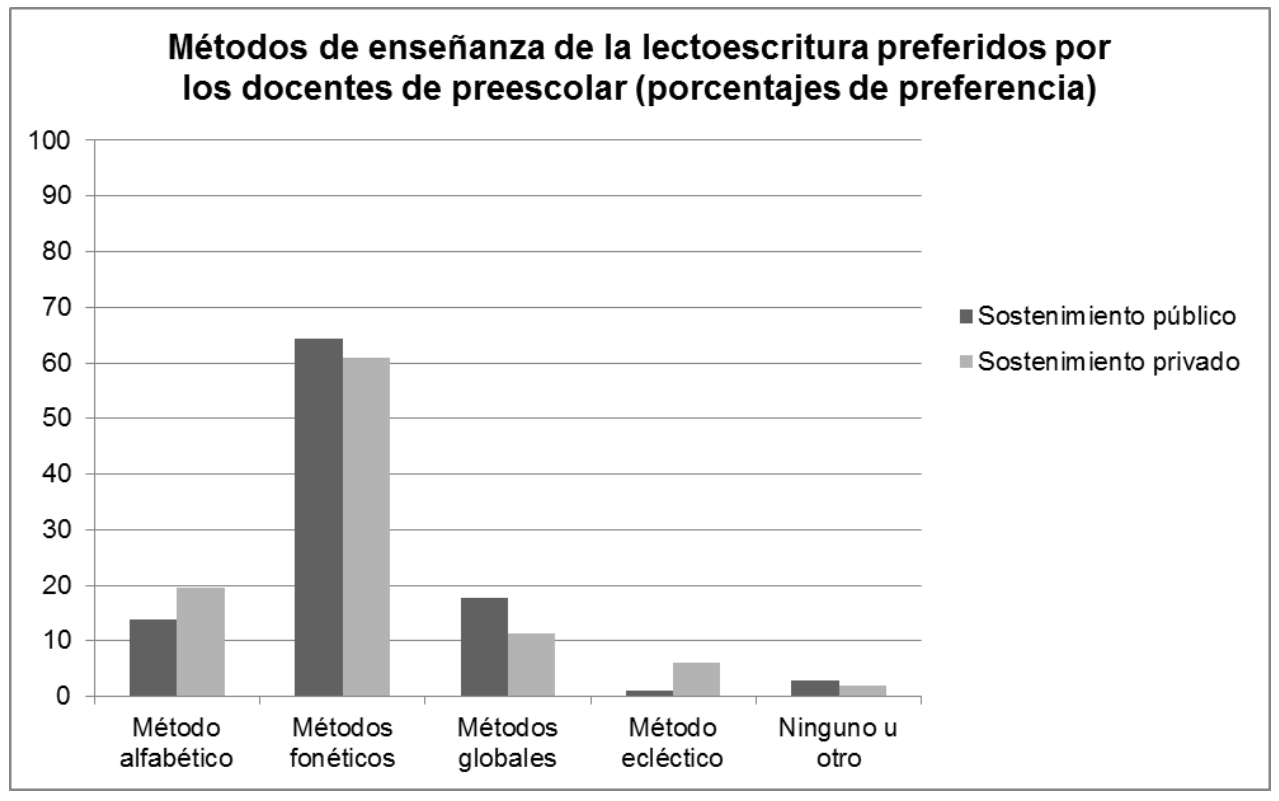

Figura 1. Métodos de enseñanza de la lectoescritura preferidos por los docentes de preescolar

La última parte de la encuesta consistía en seleccionar, de una lista de objetivos relacionados con el proceso alfabetizador, aquellos que, en la percepción del docente, le eran requeridos por los directivos de su escuela (objetivos requeridos "OR") y aquellos que el docente creía que cumpliría hacia el final del ciclo (objetivos cumplidos "OC"). Contando las menciones positivas en ambas preguntas, se calculó la razón de cumplimiento de objetivos (OC/OR), según tipo de sostenimiento de la institución del docente y el grado escolar. Por ejemplo, si un docente de tercero de un preescolar público contestó que su institución le pedía que cumpliera con el objetivo 18 (Que el niño escriba textos cortos) y cuatro docentes contestaron que creían que cumplirían ese mismo objetivo hacia el final del curso, la razón de cumplimiento 
se calculó dividiendo cuatro entre uno (4/1), lo cual dio como resultado 4. Para ese mismo objetivo, el cálculo del cumplimiento en docentes de escuelas privadas dio como resultado 0.9 (al dividir 6/7).

Para analizar el resto de los resultados, deben seguirse las siguientes consideraciones: las cantidades iguales o superiores a 1 indican que el objetivo se cumple en igual o mayor medida que la que se requiere por las autoridades escolares. Las cantidades inferiores, que el objetivo se cumple en menor medida. Si ningún docente reportó que la institución le solicitará cumplir con el objetivo correspondiente, el cálculo es indefinido. Las Figuras 2, 3, 4 y 5 muestran dichos resultados.

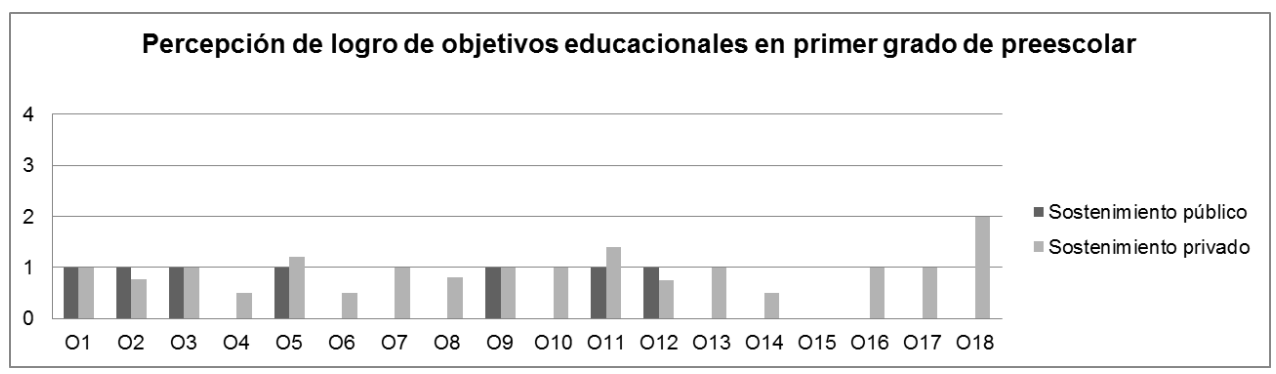

Figura 2. Percepción de logro de objetivos educacionales en primer grado de preescolar

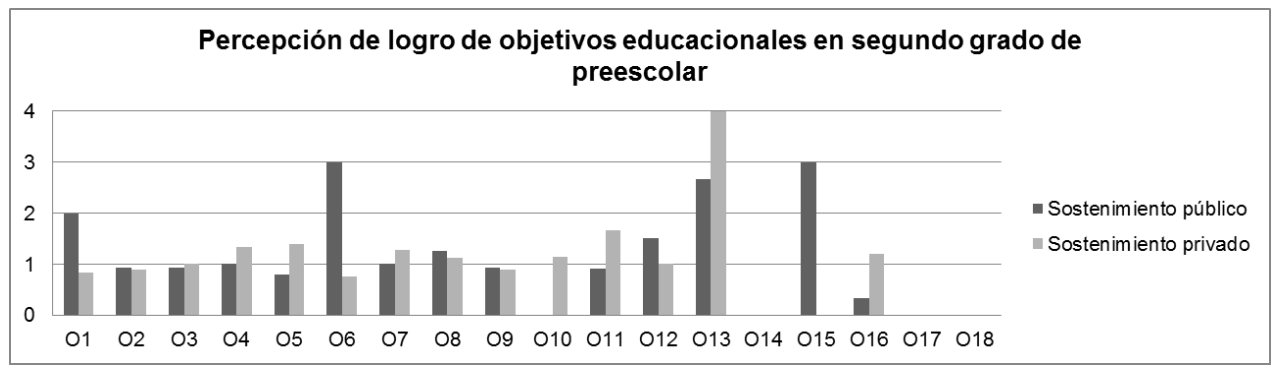

Figura 3. Percepción de logro de objetivos educacionales en segundo grado de preescolar

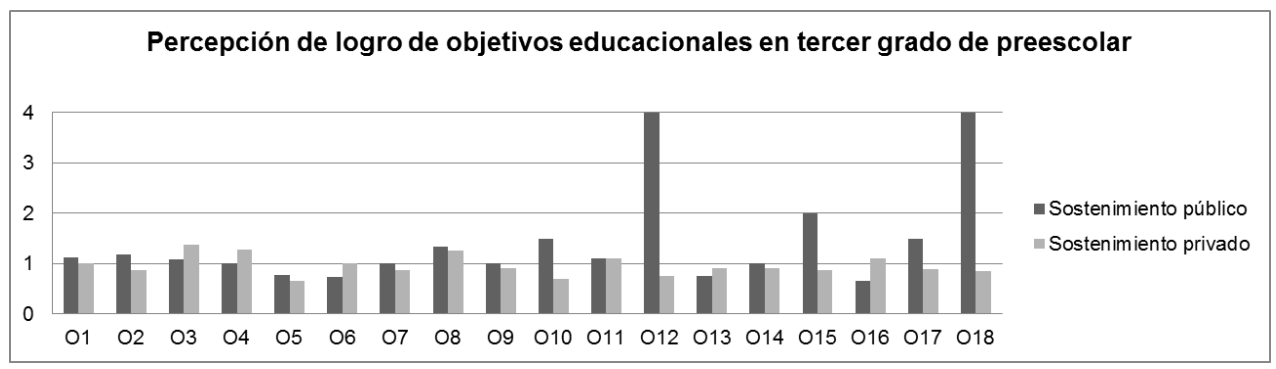

Figura 4. Percepción de logro de objetivos educacionales en tercer grado de preescolar 


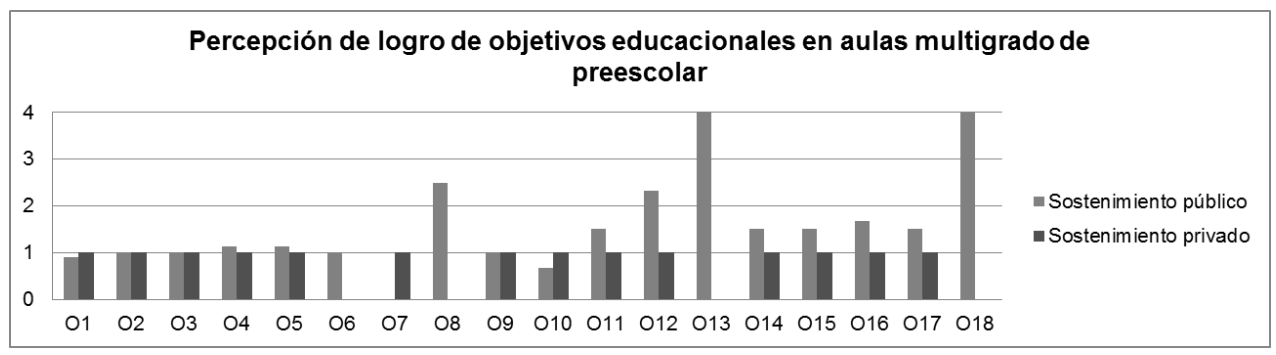

Figura 5. Percepción de logro de objetivos educacionales en aulas multigrado de preescolar

\section{Resultados del estudio cualitativo con directores de preescolar}

De acuerdo con la serie de entrevistas estructuradas a directivos de instituciones preescolares públicas y privadas, pudimos desprender que la escuela pública suele basarse totalmente en lo que solicita la Secretaría de Educación Pública en cuanto al Programa, al perfil de egreso del alumno y a sus competencias; mientras que las escuelas privadas han seguido la tendencia de ir más allá de lo que dicta: tienen sus propios objetivos educacionales, jornadas escolares y libros. Todos esos factores se ven reflejados, así mismo, en la planeación semanal del docente, por ejemplo, al serle exigido cumplir con más objetivos de los que demanda la autoridad federal -que los alumnos de preescolar salgan leyendo fluido, escribiendo, sumando y restando, entre otros-.

En la Tabla 5 se presenta un cuadro comparativo de la educación preescolar pública y la privada en México, elaborado con base en las respuestas de este instrumento.

Tabla 5. Escenarios en el logro de objetivos educacionales de lectoescritura en preescolares mexicanos

\begin{tabular}{|l|l|c|l|}
\hline \multicolumn{4}{|c|}{ Escenarios en el logro de objetivos educacionales de lectoescritura en preescolares } \\
mexicanos
\end{tabular}




\section{Discusión Y CONCLUSIONES}

Con base en los resultados, se pueden precisar algunas de las características de la educación preescolar mexicana actual. Por una parte, las escuelas públicas y las privadas se basan en el Programa vigente y en los libros publicados por la SEP; sin embargo, las escuelas privadas suelen basarse, además, en otros libros y añadir contenidos al Programa oficial. Tanto las respuestas de los directores como de los docentes coinciden en este punto.

En segundo lugar, al menos la mitad de los docentes se encuentra estudiando algún programa educativo, sea de Licenciatura, de Posgrado o de capacitación, lo cual les da la oportunidad de articular la teoría pedagógica con la práctica educativa.

Por otra parte, los escenarios a los que se enfrentan estos docentes son variados: con grupos de 4 o de 35 alumnos, deben buscar cumplir los objetivos educacionales propuestos por la autoridad federal y por su propia autoridad escolar, a veces sin ayuda de los padres de familia. Es importante destacar que, en la percepción de estos docentes, es más probable que los alumnos de escuelas privadas reciban ayuda de sus padres para el proceso alfabetizador.

Para los docentes de educación preescolar privada la dificultad aumenta. En la mayoría de los casos los objetivos requeridos por la autoridad escolar son mayores que los requeridos por la autoridad pública, por lo que es menos probable que puedan cumplir a cabalidad estos objetivos.

Si bien los límites de esta investigación no permiten confirmar la relación entre la realidad pedagógica de la escuela preescolar y su parte económica y sociopolíti$\mathrm{ca}$, podemos suponer que las cuestiones sociales, específicamente mercadotécnicas, tienen sobrada inferencia en cómo los directores o dueños de los colegios privados establecen los objetivos y los gestionan.

Toda investigación tiene sus implicaciones prácticas, y esta no es la excepción. Al conocer el escenario al que se enfrentará, el docente de este nivel puede ajustar su planeación, sus estrategias y métodos para lograr un beneficio en el niño que asiste a la institución preescolar. Esta postura es compartida por otros autores, como Ganem y Ragasol (2015), quienes afirman que "lo que han hecho los teóricos es intentar descifrar la forma en que el sujeto aprende, y eso los maestros lo traducen de manera cotidiana en estrategias concretas" (p. 11), o como Goodman (2003), quien afirma que "lo que los investigadores sabemos es importante para la enseñanza, pero quien lo sabe (el maestro) y lo que se hace con lo que sabe, es aún más importante" (p. 77).

Aclaramos, para finalizar, que los presentes resultados y conclusiones tienen un carácter eminentemente reflexivo. Es imperante incentivar en este nivel la investigación educativa en todos sus diseños, alcances y enfoques y es importante que los docentes de preescolares mexicanos reconozcan la necesidad de articular la teoría y la práctica. Solo así se podrán superar las barreras establecidas por la división de la educación en estratos. Sin importar las limitantes condiciones económicas de la sociedad y sin importar si se suele imaginar a la educación privada únicamente como 
una estrategia para el rendimiento de utilidades, puede haber educación pública y privada de calidad, siempre y cuando los docentes de este nivel pueden hacer valer su práctica docente al basarla en la investigación y la teoría.

\section{REFERENCIAS BIBLIOGRÁFICAS}

Acuerdo número 07/06/17 por el que se establece el Plan y los Programas de Estudio para la Educación Básica: Aprendizajes clave para la educación integral, Diario Oficial de la Federación: Órgano del Gobierno Constitucional de los Estados Unidos Mexicanos, Tomo DCCLXV, No. 22, Segunda Sección (2017).

Acuerdo número 348 por el que se determina el Programa de Educación Preescolar, Diario Oficial de la Federación: Órgano del Gobierno Constitucional de los Estados Unidos Mexicanos, Tomo DCXIII, No. 19, Segunda Sección (2004).

Acuerdo número 357 por el que se establecen los requisitos y procedimientos relacionados con la autorización para impartir educación preescolar, Diario Oficial de la Federación: Órgano del Gobierno Constitucional de los Estados Unidos Mexicanos, Tomo DCXXI, No. 3, Primera Sección (2005).

Acuerdo número 592 por el que se establece la Articulación de la Educación Básica, Diario Oficial de la Federación: Órgano del Gobierno Constitucional de los Estados Unidos Mexicanos, Tomo DCXCV, No. 15, Segunda Sección (2011).

Arnaz, J. A. (1990). La planeación curricular (2. ${ }^{\text {a }}$ ed.). México, D. F.: Trillas.

Braslavsky, B. (2004). ¿Primeras letras o primeras lecturas? Una introducción a la alfabetización temprana. Buenos Aires, Argentina: Fondo de Cultura Económica de Argentina.

Braslavsky, B. (2014). La querella de los métodos en la enseñanza de la lectura: Sus fundamentos psicológicos y la renovación actual. Buenos Aires, Argentina: UNIPE: Editorial Universitaria.

Chaves Salas, A. L. (2002). Procesos iniciales de lecto-escritura en el nivel de educación inicial. Actualidades Investigativas en Educación, 2(1), 1-23. http://doi. org/10.15517/aie.v2i1.8468.

Comenio, J. A. (2006). Didáctica magna (16. a ed. Trad. Saturnino López Peces). México, D.F.: Porrúa.

De-La-Peña, C., Parra-Bolaños, N. y Fernández-Medina, J. M. (2018). Análisis de la alfabetización inicial en función del tipo de familia. Ocnos, 17(1), 7-20. http://doi. org/10.18239/ocnos_2018.17.1.1336.

Ferreiro, E. (2000). Cultura escrita y educación: Conversaciones con Emilia Ferreiro. Con José Antonio Castorina, Daniel Goldin y Rosa María Torres (2. a ed.). México: Fondo de Cultura Económica.

Ganem Alarcón, P. y Ragasol Álvarez, M. C. (2015). Piaget y Vigotski en el aula: El constructivismo como alternativa de trabajo docente. México, D.F.: Limusa. 
Goodman, K. (2003). El aprendizaje y la enseñanza de la lectura y la escritura (Trad. Amparo Clavijo Olarte). Enunciación, 8, 77-98. Recuperado de https://revistas. udistrital.edu.co/ojs/index.php/enunc/article/view/2480/3463

Instituto Nacional de Estadística y Geografía (2017). Anuario estadístico y geográfico de Nuevo León 2017. México D. F.: Instituto Nacional de Estadística y Geografía.

Modelo educativo para la educación obligatoria, Diario Oficial de la Federación: Órgano del Gobierno Constitucional de los Estados Unidos Mexicanos, Tomo 765 No. 21, Primera Sección (2017).

Narodowski, M. y Martínez Boom, A. (2016). ¿Por qué se expande la educación privada?: Aportes para el debate global. Revista Colombiana de Educación, 70, 17-26. http://doi.org/10.17227/01203916.70rce17.26.

Orellana-García, P. y Melo-Hurtado, C. (2014). Ambiente letrado y estrategias didácticas en la educación preescolar chilena. Magis, 6(13), 113-128. http://doi. org/10.11144/Javeriana.M6-13.ALED.

Ramos, M. y Ramos, J. (2010). Calendario del desarrollo infantil. México: Proed Latinoamérica.

Ramos Valdez, A. (2007). Algunos protagonistas de la pedagogía: vida y obra de grandes maestros. Antología básica. México, D. F.: Universidad Pedagógica Nacional.

Rivas González, M. R. (2007). La lectura y la escritura en la escuela preescolar mexicana. Dimensión historiográfica. Revista Investigación Educativa Duranguense, 2(6), 30-47. Recuperado de http://www.upd.edu.mx/PDF/ Revistas/InvestigacionEducativaDuranguense6.pdf.

Rugerio, J. P. y Guevara, Y. (2015). Alfabetización inicial y su desarrollo desde la escuela preescolar. Ocnos: Revista de estudios sobre lectura, 13, 25-42. http://doi. org/10.18239/ocnos_2015.13.02.

Secretaría de Educación Pública (2017). Aprendizajes clave para la educación integral. Educación preescolar. México, D. F.: Secretaría de Educación Público.

Solana, F., Cardiel Reyes, R. y Bolaños, R. (Coords.) (2001). Historia de la educación pública en México (2. ${ }^{a}$ ed.). México, D. F.: Fondo de Cultura Económica.

Swartz, S. L., Shook, R., Klein, A. y Hagg, C. (2001). Enseñanza inicial de la lectura y la escritura. México, D. F.: Trillas.

Zárate González, J. (2015). La práctica de enseñanza en México de 1920-1940. En J. Arriaga Méndez, D. Castillo Careaga y L. A. Flores Bazaldúa (Coords.), Miradas de la historia de la educación básica en México (pp. 123-129). Monterrey, Nuevo León, México: Universidad Autónoma de Nuevo León. 